\title{
DIFERENTES CARACTERÍSTICAS DOS PROFESSORES EXPLICAM O GAP EDUCACIONAL ENTRE ESCOLAS URBANAS E RURAIS NO BRASIL?
}

\author{
DIFFERENT CHARACTERISTICS OF TEACHERS \\ EXPLAIN THE EDUCATIONAL GAP BETWEEN URBAN \\ AND RURAL SCHOOLS IN BRAZIL?
Nataniele dos Santos Alencar* Jair Andrade de Araujo** Wellington Ribeiro Justo*** Diogo Brito Sobreira****

\begin{abstract}
Resumo
O estudo objetiva analisar como as diferenças nas características dos professores podem explicar a lacuna no desempenho educacional dos alunos do $9^{\circ}$ ano do ensino fundamental entre escolas públicas urbanas e rurais no Brasil em 2017. Os resultados mostram que professores com licenciatura na área influenciam positivamente e significativamente o desempenho em Língua Portuguesa e Matemática, sendo o efeito maior para estudantes de escolas rurais. Contudo, essa influência descresse à
\end{abstract}

dos Santos Alencar, N., Andrade de Araujo, J., Ribeiro Justo, W., \& Brito Sobreira, D. (2021). Diferentes características dos professores explicamo gap educacional entre escolas urbanas e rurais no Brasil? Estudios económicos, 38(76), 45-68.

* Universidade Federal do Ceará, Brasil. E-mail: jaraujoce@gmail.com. ORCID: https://orcid. org/0000-0001-6297-9558

** Universidade Federal do Ceará, Brasil. E-mail: jaraujoce@gmail.com. ORCID: https://orcid. org/0000-0001-6297-9558

*** Universidade Regional do Cariri, Brasil. E-mail: justowr@yahoo.com.br. ORCID: https://orcid. org/0000-0002-4182-4466

**** Universidade Federal do Ceará, Brasil. E-mail: diogobsobreira@gmail.com. ORCID: https:/orcid. org/0000-0002-6840-754X 
medida que aumenta o quantil em ambas as áreas. Ademais, a decomposição sugere que a contribuição agregada das diferenças nas características dos professores, e de seus coeficientes, sobre os diferenciais é pequena quando comparada ao efeito agregado das outras características.

Palavras-chave: Diferenças Educacionais; Áreas Urbanas e Rurais; Prova Brasil. Classificação JEL: I24, I25, J24, C51.

\begin{abstract}
The study aims to analyze how differences in teacher characteristics may explain the gap in educational performance of 9 th grade students between urban and rural public schools in Brazil in 2017. The results show that teachers with a degree in area that teaches positively and significantly influence the performance in Portuguese Language and Mathematics, the effect being greater for students of rural schools. However, this influence declined as the quantile in both areas increased. Moreover, the decomposition suggests that the aggregate contribution of differences in teacher characteristics, and their coefficients, to differentials is small compared to the aggregate effect of other characteristics.
\end{abstract}

Keywords: Educational Differences; Urban and Rural Areas; Prova Brasil. JEL Codes: I24, I25, J24, C51. 


\section{INTRODUÇÃO}

O sistema educacional brasileiro ${ }^{1}$ é apontado como um dos principais responsáveis para o baixo grau de desenvolvimento do país, e a qualidade da educação básica ainda está abaixo dos padrões internacionais (Pessôa \& Barbosa Filho, 2008). Além disso, o acesso e a qualidade do ensino ainda são desiguais, principalmente para os grupos populacionais desfavorecidos, como os estudantes de escolas rurais. Os resultados da última Prova Brasil, em 2017, revelam que os estudantes de escolas rurais do $9^{\circ}$ ano do ensino fundamental estão atrasados em um nível da escala de proficiência em relação aos de escolas urbanas².

Diante das diferenças existentes na educação básica brasileira, existem insumos comuns a todos os níveis educacionais, como as características dos professores que estão diretamente associadas ao processo de aprendizado dos estudantes. O INEP (2020) revela um crescimento na proporção dos professores com nível superior nos anos finais do ensino fundamental público, passando de $82,5 \%$ para $92,7 \%$, entre 2011 e 2019. Contudo, a proporção de docentes com ensino superior em áreas urbanas e rurais permanece desigual ${ }^{3}$.

Evidências como essas fomentam vários questionamentos, mas, o presente estudo centra-se em duas: (i) as diferenças nas características dos professores são capazes de explicar as diferenças de desempenho entre as duas áreas? (ii) essa influência é padrão entre os estudantes com distintos níveis de proficiência?

Assim, o artigo tem como objetivo analisar como as diferenças nas características dos professores contribuem para explicar o gap de desempenho dos estudantes do $9^{\circ}$ ano de escolas públicas urbanas e rurais brasileiras. Esse tema,

1 O sistema está dividido em ensino básico (educação infantil, ensino fundamental, ensino médio) e ensino superior, em que a oferta do ensino básico é majoritariamente pública ( $81 \%$ das matrículas em 2019), cujo investimento público é financiado, principalmente, pelo Fundeb. A educação infantil e o ensino fundamental são de responsabilidade da gestão municipal, enquanto o ensino médio é designado aos estados e o ensino superior ao governo federal. A oferta de ensino em áreas rurais no Brasil é quase que totalmente pública. Em 2019, 99\% das matrículas nos anos finais do ensino fundamental em escolas rurais foram realizadas no ensino público.

2 Em 2017, o desempenho de estudantes de escolas urbanas foi de 253 e 251 pontos em Língua Portuguesa e Matemática, respectivamente, que corresponde ao terceiro nível na escala de proficiência. Já os de escolas rurais realizaram 235 pontos em ambas as avaliações.

3 Por exemplo, em 2011, a proporção de professores com ensino superior em escolas públicas urbanas nos anos finais do ensino fundamental era de 91\% e em áreas rurais era de 54,7\%. Em 2019 essas proporções aumentam para 97\% e 78,4\%, respectivamente (INEP, 2020). 
entretanto, tem sido amplamente debatido na literatura, tanto para países em desenvolvimento (Tayyaba, 2011; Lounkaew, 2013; Ramos, Duque \& Sandra Nieto, 2016; Wang, Li \& Wang, 2018 e Rodrigues, Costa, Miro, Mariano \& Jesus Filho, 2018) quanto em países desenvolvidos (Byun, Meece \& Irvin, 2012).

Neste estudo, adotou-se o procedimento proposto por Firpo, Fortin \& Lemieux (2018), que permite obter a contribuição detalhada nos componentes, explicado e não explicado, da decomposição Oaxaca-Blinder nos quantis de distribuição de desempenho dos estudantes. Assim, o presente estudo contribui para a literatura ao fornecer estimativas de como as diferenças nas características dos professores explicam o gap educacional entre estudantes de escolas urbanas e rurais para estatísticas que vão além da média dos dados. Além disso, a estratégia adotada supera fatores limitantes de outras abordagens ${ }^{4}$

No caso brasileiro, Rodrigues et al. (2018) adotaram a mesma estratégia empírica para verificar os determinantes dos diferenciais educacionais de estudantes no $5^{\circ}$ ano do fundamental. Contudo, é possível que a influência das características dos professores não seja a mesma sobre estudantes em diferentes níveis de ensino. Desse modo, outra contribuição deste estudo é fornecer uma análise dos diferenciais de desempenho para estudantes do $9^{\circ}$ ano do ensino fundamental (último grau de ensino para garantir o acesso ao ensino médio no Brasil). Adicionalmente, características dos professores que não foram consideradas em estudos anteriores ${ }^{5}$ foram incorporadas em nossas estimativas, como a formação superior na área em que o professor leciona.

Portanto, em função da influência que o professor exerce sobre o desempenho dos alunos, além de outras disparidades socioeconômicas aos quais os estudantes de áreas urbanas e rurais estão expostos, faz-se necessário uma maior atenção por parte dos pesquisadores e policy markers, especialmente em países em desenvolvimento, onde a desigualdade de oportunidades é maior.

O artigo é composto por mais quatro seções, além desta introdução. A segunda seção apresenta a revisão de literatura; na terceira são apresentados os procedimentos metodológicos; na quarta, os resultados e discussões; por fim, temse as considerações finais.

4 Al-Samarrai \& Reilly (2000); Ramos, Duque \& Sandra Nieto (2016), Cuesta (2018) realizam decomposições para média dos dados. Já Sakellariou (2012) faz uso de regressões quantílicas condicionais para decompor os diferenciais.

5 Lounkaew (2013) e Rodrigues et al. (2018). 


\section{REVISÃO DE LITERATURA}

Para explicar a importância dos professores, definiu/define uma Função de Produção Educacional considerando também outros insumos educacionais. Nesse sentido, as características dos estudantes e da família são importantes fatores associados ao desempenho dos estudantes. Já, Hammond (2000) e enfatizam a influência das características da escola e dos professores (tamanho da classe, localização, tipo da escola, qualificação dos docentes, experiência dos professores, salário dos professores, recursos educacionais).

Sobre o papel dos professores, resultados diversos são encontrados na literatura. Menezes-Filho e Pazello (2007), por exemplo, mostram que o aumento salarial dos professores na rede pública permitiu ganhos significantes sobre o desempenho dos estudantes no Brasil. Clotfelter, Ladd \& Vigdor (2007) encontram efeitos positivos da experiência dos professores e da licenciatura regular. Por outro lado, títulos de pós-graduação parecem não tornar os professores mais eficazes sobre o desempenho de estudantes nos EUA. Tais evidências, entretanto, vão de encontro aos resultados de Krueger (1999). Por meio de um experimento randomizado, o estudo mostra que as características dos professores (por exemplo, raça, sexo, experiência e pós-graduação) têm pouca importância sobre o desempenho dos estudantes.

Além dos fatores que afetam o desempenho dos estudantes, as disparidades de desempenho entre estudantes urbanos e rurais têm sido investigadas na literatura recente. Diversos estudos mostram que os alunos de escolas em áreas rurais têm os piores resultados educacionais (Roscigno, Tomaskovic-Devey \& Crowley, 2006; Strayhorn, 2009; Ramos, Duque \& Nieto, 2016). Conforme destacam Roscigno \& Crowley (2001), o baixo desempenho desses estudantes está relacionado à falta de recursos das famílias rurais e itens educacionais domésticos, além das baixas expectativas quanto às oportunidades de trabalho no meio rural, por parte dos pais e dos professores em escolas rurais.

Fatores que explicam o gap entre as duas áreas, entretanto, podem variar conforme o sistema educacional ou nível de ensino considerado. No Paquistão, por exemplo, Tayyaba (2012) destaca que as condições de escolaridade, origem dos alunos e o treinamento dos professores são decisivos para determinar o gap. Ramos, Duque \& Nieto (2016) mostram, por métodos de decomposição, que a maior parte do diferencial é atribuível às diferenças nas características da família. 
No caso brasileiro, Rodrigues et al. (2018) destacam que as diferenças nas características da escola respondem por maior parte das diferenças entre estudantes do $5^{\circ}$ ano. Por outro lado, Rodrigues, Costa, Miro \& Mariano (2017) encontram que a diferença de desempenho no ensino médio brasileiro é favorável aos estudantes urbanos, mesmo quando comparada a estudantes de áreas rurais que estudam em escolas urbanas, sendo explicada principalmente pelas diferenças nas características individuais e familiares. Neste estudo, os diferenciais entre estudantes de escolas urbanas e rurais no Brasil, entretanto, foram analisados para estudantes do $9^{\circ}$ ano do ensino fundamental da rede pública. Além disso, dada a importância dos professores no processo educacional, este estudo analisa como a diferença de características dos professores, entre os dois tipos de escolas, pode explicar as diferenças de desempenho entre as duas áreas.

\section{ESTRATÉGICA EMPÍRICA}

O presente estudo tem como objetivo analisar como as diferenças nas características dos professores, e nos coeficientes, podem explicar o diferencial educacional entre estudantes de escolas urbanas e rurais, em diferentes níveis de desempenho. Desse modo, funções de produção educacional (FPE) foram estimadas para cada grupo separadamente, tal como na equação (1):

$$
Y_{i t}=X_{i t} \beta_{t}+e_{i t}, T=\{1=\text { urbano, } 0=\text { rural }\}
$$

em que, $Y_{i t}$ é o logaritmo natural do desempenho do estudante $i$ em Português ou Matemática no grupo $t ; X_{i}$ é o vetor de características explicativas; $\beta_{t}$ é o vetor de coeficientes do grupo $t$; $e_{i t}$ é o erro idiossincrático.

Métodos de decomposição Oaxaca-Blinder (1973), aplicadas às regressões dos dois grupos, tal como na equação (2), permitem realizar a decomposição agregada dos diferenciais totais em dois componentes (equação 3):

$$
\begin{gathered}
\Delta=\bar{Y}_{1}-\bar{Y}_{0}=\bar{X}_{1} \hat{\beta}_{1}-\bar{X}_{0} \hat{\beta}_{0} \\
\Delta=\Delta_{x}+\Delta_{s}=\left(\bar{X}_{1}-\bar{X}_{0}\right) \hat{\beta}_{1}+\bar{X}_{0}\left(\hat{\beta}_{1}-\hat{\beta}_{0}\right)
\end{gathered}
$$


em que, $\left(\bar{X}_{1}-\bar{X}_{0}\right) \hat{\beta}_{1}$ é a parcela do diferencial que é explicada pelas diferenças nas características (componente explicado) e $\bar{X}_{0}\left(\hat{\beta}_{1}-\hat{\beta}_{0}\right)$ é a parcela explicada pela diferença nos coeficientes (componente não explicado). Ademais, uma decomposição detalhada dentro desses componentes também pode ser obtida. Desse modo, pode-se estimar a contribuição específica do conjunto das características dos professores sobre o gap educacional entre as duas áreas.

Neste estudo, entretanto, adotou-se uma extensão desse método que combina regressões-RIF (Recentred Influence Funtion) e uma abordagem de reponderação ${ }^{6}$ para realizar decomposições detalhadas, conforme Firpo, Fortin \& Lemieux (2018). O método permite realizar a decomposição detalhada dos diferenciais para estatísticas que vão além da média 7 . Desse modo, regressões quantílicas incondicionais (regressões-RIF nos quantis) foram estimadas e, em seguida, utilizadas na decomposição dos diferenciais em diferentes níveis de desempenho dos estudantes.

O método é realizado em dois estágios. No primeiro, os pesos são computados para reponderar a área de interesse de modo a obter uma distribuição contrafactual $F_{Y}^{C}$ :

$$
\hat{\omega}_{1}(T)=\frac{T}{\hat{p}} \hat{\omega}_{0}(T)=\frac{1-T}{1-\hat{p}} \hat{\omega}_{c}(T, X)=\frac{(1-T)}{\hat{p}} \cdot \frac{\hat{p}(X)}{1-\hat{p}(X)}
$$

em que, $T$ indica a que grupo o $i$-ésimo estudante pertence, 0 ou $1 \mathrm{e}$ $\hat{p}=N^{-1} \sum_{i=1}^{N} T_{i} . \hat{p}(X)$ é a probabilidade de pertencer ao grupo 1 dado $X$. Os pesos são utilizados para obter as distribuições acumuladas de cada grupo $T$ e a distribuição contrafactual:

$$
\hat{F}_{T}(y)=\sum_{i=1}^{N} \hat{\omega}_{t}\left(T_{i}\right) . l\{Y \leq y\}, t=0,1
$$

6 Como em Dinardo, Fortin \& Lemieux (1996).

7 Algumas abordagens não realizam a decomposição detalhada em diferentes pontos da distribuição no componente não explicado e são path-dependence. Ver Fortin, Lemieux \& Firpo (2011) para consultar as limitações e vantagens dos métodos de decomposição. 


$$
\hat{F}_{c}(y)=\sum_{i=1}^{N} \hat{\omega}_{c}\left(T_{i}, X_{i}\right) . l\{Y \leq y\}
$$

O segundo estágio é utilizado para calcular a contribuição detalhada em ambos componentes. Os autores usam regressões- $R I F$, em que a variável de resultado, $Y$, é substituída pela função de influência recentrada, permitindo a estimação do efeito das mudanças de um conjunto de covariadas $X$ na estatística $v\left(F_{y}\right)$ da distribuição marginal de $Y$. Primeiro, o cálculo da $R I F$ é realizado para os quantis, como na equação (6):

$$
R \hat{I I}\left(y ; q_{\tau}, F_{y}\right)=\hat{q}_{\tau}+\frac{\tau-I\left\{y \leq \hat{q}_{\tau}\right\}}{\hat{f}_{y}\left(\hat{q}_{\tau}\right)}
$$

em que, $\hat{q}_{\tau}$ é o quantil e o segundo termo da soma é a função de influência. $I\left\{y \leq \hat{q}_{\tau}\right\}$ é uma função indicador que é menor ou igual ao $q_{\tau}$, e $\hat{f}_{y}\left(\hat{q}_{\tau}\right)$ é uma função de densidade da distribuição marginal de $y$ estimada por Gaussian kernel function ${ }^{8}$ e avaliada em $q_{\tau}$.

Substituindo os valores de $Y_{i}$ da equação (1) pelos valores de obtidos em $R \hat{I F}\left(y ; q_{\tau}, F_{y}\right)(6)$, regressões quantílicas para cada grupo $T$ podem ser estimadas por mínimos quadrados ordinários, tal como a equação (7):

$$
E\left[R \hat{I F}\left(y_{i}, q_{\tau, t}, F_{y, t}\right) \mid X_{i}\right]=X_{i} \hat{\gamma}_{t}^{q_{\tau}}, t=0,1
$$

em que, $\hat{\gamma}^{q_{\tau}}$ é o efeito na mudança no valor médio de $X$ sobre o quantil incondicional de $Y$. Utilizando os pesos, os parâmetros $\hat{\gamma}_{t}^{q_{\tau}}$ podem ser estimados para cada grupo $T$ e contrafactual (c) da seguinte forma:

$$
\begin{gathered}
\hat{\gamma}_{t}^{q_{\tau}}=\left(\sum_{i=1}^{N} \hat{\omega}_{t}\left(T_{i}\right) X_{i} X_{i}^{\prime}\right)^{-1} \cdot \sum_{i=1}^{N} \hat{\omega}_{t}\left(T_{i}\right) X_{i} R \hat{I} F\left(y_{i} ; q_{\tau, t}, F_{y, t}\right), t=0,1 \\
\hat{\gamma}_{c}^{q_{\tau}}=\left(\sum_{i=1}^{N} \hat{\omega}_{c}\left(T_{i}, X_{i}\right) X_{i} X_{i}^{\prime}\right)^{-1} \cdot \sum_{i=1}^{N} \hat{\omega}_{c}\left(T_{i}, X_{i}\right) X_{i} R \hat{I} F\left(y_{i} ; q_{\tau, c}, F_{y, c}\right)
\end{gathered}
$$

8 Estimada com valor ótimo de half-width. 
O resultado da decomposição aplicada ao procedimento de reponderação é dada por (10):

$$
\hat{\Delta}^{q_{\tau}}=\hat{\Delta}_{X, p}^{q_{\tau}}+\hat{\Delta}_{X, e}^{q_{\tau}}+\hat{\Delta}_{S, p}^{q_{\tau}}+\hat{\Delta}_{S, e}^{q_{\tau}}
$$

em que $\hat{\Delta}_{X, p}^{q_{\tau}}$ é o efeito explicado puro obtido por meio da amostra $\mathrm{T}=0$ e a contrafactual que é $\mathrm{T}=0$, reponderada como em $\mathrm{T}=1$, usando $\mathrm{T}=0$ como estrutura de referência. $\hat{\Delta}_{X, e}^{q_{\tau}}$ é o erro de especificação ou aproximação $\hat{\Delta}_{S, p}^{q_{\tau}}$ é o efeito não explicado puro. $\hat{\Delta}_{S, e}^{q_{\tau}}$, é o erro de reponderação que tende a zero em grandes amostras

O efeito detalhado dos coeficientes atribuído às variáveis dummy não é invariante para a escolha dos grupos de referência. Assim, esse componente pode sofrer com problemas de identificação. Para solucionar essa questão, adotou-se o procedimento de Yun (2005).

\section{II.1. Base de dados}

Para alcançar os objetivos, foram utilizados os microdados da Prova BrasilSAEB de 2017, dos alunos do $9^{\circ}$ ano do ensino fundamental da rede pública, disponibilizada pelo INEP. Os desempenhos dos estudantes, em logaritmo natural, em Língua Portuguesa e Matemática foram utilizados como variáveis de resultado. As variáveis explicativas incluídas no modelo podem ser divididas em cinco grupos de características:

1. Estudante: dummy de Sexo, que assume valor 1 para masculino e 0, se feminino; dummy de Cor, que assume valor 1 para brancos e 0, para outras; Idade, número de meses na data da prova (outubro de 2017) transformados em anos; e três dummies: Reprovação, Atividades feitas em Casa e Trabalho, que assumem valor 1 para ocorrência do evento e 0 , caso contrário.

2. Familiar: duas dummies, Computador em casa e Incentivo à frequência escolar, que assumem valor 1 se os eventos ocorrem e 0, caso contrário; dummies para escolaridade da mãe e escolaridade do pai (fundamental incompleto, fundamental completo, médio completo e superior completo), que assumem valor 1 se os pais possuem o grau de escolaridade e 0 , caso contrário;

3. Escola: duas dummies para existência de Biblioteca e Laboratório de informática, que recebem valor para existência e 0 , caso contrário; Índice de Nível 
Socioeconômico (INSE) ${ }^{9}$, variável categórica com valores: 1) muito baixo, 2) baixo, 3) médio baixo, 4) médio, 5) médio alto e 6) alto.

4 Professores: dummy de Sexo, que assume valor 1 para professores do sexo masculino e 0 , caso contrário; dummy de Cor, que recebe valor 1 para aqueles que se declaram brancos e 0 , para outras cores; variável categórica que representa a Faixa etária dos professores que recebe os valores: 1 (até 24 anos), 2 (25 a 39 anos), 3 (40 a 54 anos) e 4 (55 ou mais); dummies para Licenciatura na área que leciona, Ausência de pós-graduação, Carência de infraestrutura ${ }^{10}$, Alto indice de faltas, que recebem valor 1 para ocorrência dessa característica e 0 , caso contrário; e, quatro dummies para as faixas salariais ( 1 a 2 salários, 2 a 3 salários, 3 a 5 salários e 5 ou mais salários).

5. Regionais: cinco dummies para as regiões Norte, Nordeste, Sudeste, Sul e Centro-Oeste.

A Prova Brasil possui informações por escolas de áreas urbanas e rurais brasileiras. Foi usado o filtro pela variável IN_PROVA_BRASIL, a qual indica se o aluno participou ou não da Prova Brasil. Aplicada a cada dois anos, os alunos são avaliados nas disciplinas de Língua Portuguesa e Matemática.

\section{RESULTADOS E DISCUSSÕES}

\section{III.1. Análise descritiva}

Essa seção destaca as estatísticas descritivas ${ }^{11}$. Os resultados mostram que estudantes de escolas urbanas têm melhores desempenhos que os de escolas rurais em ambas as disciplinas, como em avaliações anteriores (Rodrigues et al., 2017, 2018). Em Língua Portuguesa, a média urbana foi de 262 e 244 para escolas rurais. Já em Matemática, as médias foram, respectivamente, 260 e 244 pontos. Essas diferenças evidenciam níveis de proficiência distintos entre os dois meios, em média.

9 Padrão de vida dos estudantes da escola, com base na escolaridade dos pais, da posse de bens e contratação de serviços pela família dos alunos.

10 Carência de infraestrutura e índice de falta refletem a percepção dos professores quanto aos possíveis problemas para aprendizagem dos alunos.

11 Estimativas podem ser enviadas mediante solicitação. 
As evidências iniciais apontam que existem diferenças estatisticamente significantes nos atributos dos estudantes das duas áreas, que podem explicar as diferenças observadas de desempenho. Assim, observa-se que estudantes de escolas urbanas são beneficiados com mais dos insumos que influenciam positivamente o desempenho educacional. Testes de diferenças de médias entre os grupos revelam diferenças estatisticamente significantes ao nível de $5 \%$.

\section{III.2. Análise empírica}

Para avaliar a contribuição das características dos professores sobre as disparidades de desempenho entre estudantes de escola urbanas e rurais, foram estimadas regressões quantílicas incondicionais separadas para cada grupo, sendo o efeito dessas características controladas por outros insumos educacionais (características dos estudantes, família e escola).

A Tabela 1 reporta as estimativas da FPE para estudantes de escolas urbanas e rurais, e para os quantis incondicionais 10, 50 e 90 do desempenho em Língua Portuguesa ${ }^{12}$. Desse modo, é possível observar como cada característica pode influenciar as pontuações dos estudantes com baixo, mediano e alto desempenho. Grande parte das características consideradas no modelo exprimem efeitos estatisticamente significantes a $1 \%$ para as duas disciplinas. Estimativas para o desempenho em Matemática estão disponíveis na Tabela 2.

Os resultados mostram que algumas características dos estudantes estão negativamente associadas com o desempenho em Português, por exemplo, sexo masculino, idade, ter reprovado e trabalhar. Para estudantes de baixo desempenho em escolas rurais, ter a cor branca também influência negativamente. Para todas essas características, os efeitos aumentam no decorrer dos quantis, isto é, estudantes com alto desempenho são menos impactados pelo efeito negativo. Contudo, efeito negativo é mais intensivo para estudantes em escolas rurais do que em escolas urbanas. Os efeitos distintos entre os diferentes níveis de desempenho e entre os dois tipos de escolas justifica a análise por quantil.

Resultados semelhantes foram encontrados para o desempenho em Matemática, exceto para o sexo. Os estudantes do sexo masculino tendem a atingir melhores resultados em Matemática, enquanto que os estudantes do sexo feminino

12 Os coeficientes de algumas variáveis foram omitidos propositalmente por questões de espaço e podem ser disponibilizadas mediante solicitação. 
apresentam maior desempenho em Língua Portuguesa. Esse estudo corrobora evidências apontadas por trabalhos anteriores (Andrade, Franco \& Carvalho, 2003; Menezes-Filho \& Pazello, 2007).

Já as características familiares possuem uma influência positiva sobre os resultados educacionais dos alunos, na maioria das situações. O efeito de ter computador em casa é maior para os alunos do meio rural no quantil mediano e 90 . A influência de mães com qualificação superior, em relação às mães com ensino fundamental incompleto, torna-se mais expressiva para alunos com alto desempenho. O efeito passa a ser mais importante para alunos de escolas rurais nesse quantil. Já para os pais com ensino superior, a influência significativa, que é progressiva nos quantis, existe apenas para estudantes de escolas urbanas. ${ }^{13}$

Escolas com biblioteca e laboratório de informática estão associadas a maiores desempenhos dos alunos. Embora os efeitos dessas características decresçam nos quantis, eles são mais expressivos para escolas rurais. Ademais, o nível socioeconômico (INSE) também influência positivamente o desempenho dos estudantes. Esse resultado está de acordo com Alves \& Soares (2013), que afirmam que escolas com alunos de baixos níveis socioeconômicos, têm piores resultados educacionais.

Quanto às características dos professores, algumas características exprimem uma relação negativa sobre o desempenho em Português, por exemplo, professores homens, mudança para maiores faixas de idade, não ter pós-graduação e a percepção quanto à carência de infraestrutura das escolas e das faltas dos alunos como problemas para o desempenho educacional. A ausência de pós-graduação possui efeitos negativos maiores para os alunos de escolas rurais, porém, a intensidade desse efeito se reduz no decorrer dos quantis.

Tabela 1. FPE por quantis e área. Português - 2017

\begin{tabular}{lcccccc}
\hline & \multicolumn{2}{c}{ Q10 } & \multicolumn{2}{c}{ Q50 } & \multicolumn{2}{c}{ Q90 } \\
\hline \multirow{2}{*}{ Sexo } & Urbano & Rural & Urbano & Rural & Urbano & Rural \\
& $-0.07^{* * *}$ & $-0.06^{* * *}$ & $-0.03^{* * *}$ & $-0.04^{* * *}$ & $-0.01^{* * *}$ & $-0.017^{* * *}$ \\
\multirow{3}{*}{ Idade } & $(0.001)$ & $(0.004)$ & $(0.001)$ & $(0.003)$ & $(0.001)$ & $(0.003)$ \\
& $-0.04^{* * *}$ & $-0.03^{* * *}$ & $-0.012^{* * *}$ & $-0.02^{* * *}$ & $-0.001^{* *}$ & $-0.005^{* * *}$ \\
& $(0.001)$ & $(0.004)$ & $(0.001)$ & $(0.002)$ & $(0.0004)$ & $(0.001)$
\end{tabular}

13 Lounkaew (2013) destaca que, quanto maior a escolaridade dos pais, maior será o tempo disponível dedicado aos filhos. 


\begin{tabular}{|c|c|c|c|c|c|c|}
\hline Mãe FC & $\begin{array}{c}0.009^{* * * *} \\
(0.002)\end{array}$ & $\begin{array}{c}0.006 \\
(0.006)\end{array}$ & $\begin{array}{c}0.009^{* * *} \\
(0.001)\end{array}$ & $\begin{array}{l}0.008^{* *} \\
(0.004)\end{array}$ & $\begin{array}{l}0.002^{* *} \\
(0.001)\end{array}$ & $\begin{array}{l}0.009^{* *} \\
(0.004)\end{array}$ \\
\hline \multirow[t]{2}{*}{ Mãe MC } & $0.026^{* * *}$ & $0.016^{* * *}$ & $0.025^{* * *}$ & $0.021^{* * *}$ & $0.012^{* * *}$ & $0.021^{* * *}$ \\
\hline & $(0.002)$ & $(0.006)$ & $(0.001)$ & $(0.004)$ & $(0.001)$ & $(0.004)$ \\
\hline \multirow[t]{2}{*}{ Mãe SC } & $0.005^{* *}$ & 0.008 & $0.025^{* * *}$ & $0.011^{* *}$ & $0.026^{* * *}$ & $0.030^{* * *}$ \\
\hline & $(0.002)$ & $(0.009)$ & $(0.001)$ & $(0.006)$ & $(0.001)$ & $(0.006)$ \\
\hline \multirow[t]{2}{*}{ Biblioteca } & $0.004^{* *}$ & $0.013^{* * *}$ & $0.008^{* * *}$ & $0.012^{* * *}$ & $0.004^{* * *}$ & $0.008^{* * *}$ \\
\hline & $(0.002)$ & $(0.005)$ & $(0.001)$ & $(0.003)$ & $(0.001)$ & $(0.003)$ \\
\hline \multirow[t]{2}{*}{ Laboratório } & $0.012^{* * *}$ & $0.016^{* * *}$ & $0.007^{* * *}$ & $0.013^{* * *}$ & $0.002^{* * *}$ & $0.010^{* * *}$ \\
\hline & $(0.002)$ & $(0.004)$ & $(0.001)$ & $(0.003)$ & $(0.001)$ & $(0.003)$ \\
\hline \multirow[t]{2}{*}{ Inse } & $0.028^{* * *}$ & $0.026^{* * *}$ & $0.027^{* * *}$ & $0.026^{* * *}$ & $0.021^{* * *}$ & $0.014^{* * *}$ \\
\hline & $(0.001)$ & $(0.003)$ & $(0.001)$ & $(0.002)$ & $(0.001)$ & $(0.002)$ \\
\hline \multirow[t]{2}{*}{ Sexo prof. } & 0.0004 & $-0.010^{*}$ & $-0.004^{* * *}$ & $-0.01^{* *}$ & $-0.002^{*}$ & -0.004 \\
\hline & $(0.002)$ & $(0.006)$ & $(0.001)$ & $(0.003)$ & $(0.001)$ & $(0.003)$ \\
\hline \multirow[t]{2}{*}{ Faixa etária } & $-0.01^{* * *}$ & 0.002 & $-0.01^{* * *}$ & $-0.01^{* *}$ & $-0.01^{* * *}$ & $-0.007^{* * *}$ \\
\hline & $(0.001)$ & $(0.004)$ & $(0.001)$ & $(0.002)$ & $(0.001)$ & $(0.002)$ \\
\hline \multirow[t]{2}{*}{ Cor prof. } & $0.012^{* * *}$ & $0.019^{* * *}$ & $0.007^{* * *}$ & $0.018^{* * *}$ & $0.004^{* * *}$ & $0.014^{* * *}$ \\
\hline & $(0.002)$ & $(0.005)$ & $(0.001)$ & $(0.003)$ & $(0.001)$ & $(0.003)$ \\
\hline \multirow[t]{2}{*}{ Lic. área } & $0.006^{* *}$ & $0.023^{* * *}$ & $0.004^{* * *}$ & $0.013^{* * *}$ & $0.003^{* * *}$ & $0.006^{*}$ \\
\hline & $(0.003)$ & $(0.006)$ & $(0.001)$ & $(0.004)$ & $(0.001)$ & $(0.003)$ \\
\hline \multirow[t]{2}{*}{ Não tem pós } & $-0.01^{* * *}$ & $-0.02^{* * *}$ & $-0.004^{* * *}$ & $-0.01^{* * *}$ & $-0.01^{* * *}$ & -0.004 \\
\hline & $(0.002)$ & $(0.005)$ & $(0.001)$ & $(0.003)$ & $(0.001)$ & $(0.003)$ \\
\hline \multirow[t]{2}{*}{ Infraestrutura } & $-0.02^{* * *}$ & $-0.01^{* *}$ & $-0.014^{* * *}$ & $-0.01^{* * *}$ & $-0.01^{* * *}$ & $-0.007^{* * *}$ \\
\hline & $(0.002)$ & $(0.004)$ & $(0.001)$ & $(0.003)$ & $(0.001)$ & $(0.003)$ \\
\hline \multirow[t]{2}{*}{ Nordeste } & $-0.02^{* * *}$ & 0.009 & $0.013^{* * *}$ & $0.022^{* * *}$ & $0.016^{* * *}$ & $0.019^{* * *}$ \\
\hline & $(0.003)$ & $(0.006)$ & $(0.001)$ & $(0.004)$ & $(0.001)$ & $(0.003)$ \\
\hline \multirow[t]{2}{*}{ Sudeste } & $-0.01^{* * *}$ & 0.010 & $0.021^{* * *}$ & $0.050^{* * *}$ & $0.016^{* * *}$ & $0.050^{* * *}$ \\
\hline & $(0.003)$ & $(0.008)$ & $(0.001)$ & $(0.005)$ & $(0.001)$ & $(0.005)$ \\
\hline \multirow[t]{2}{*}{ Sul } & $0.018^{* * *}$ & 0.008 & $0.021^{* * *}$ & $0.025^{* * *}$ & 0.002 & 0.008 \\
\hline & $(0.003)$ & $(0.010)$ & $(0.002)$ & $(0.007)$ & $(0.001)$ & $(0.008)$ \\
\hline \multirow[t]{2}{*}{ Centro-Oeste } & $0.026^{* * *}$ & $0.044^{* * *}$ & $0.03^{* * *}$ & $0.048^{* * *}$ & $0.008^{* * *}$ & $0.021^{* * *}$ \\
\hline & $(0.003)$ & $(0.009)$ & $(0.001)$ & $(0.007)$ & $(0.001)$ & $(0.007)$ \\
\hline \multirow[t]{2}{*}{ Constante } & $5.531^{* * *}$ & $5.369^{* * *}$ & $5.5797^{* * *}$ & $5.615^{* * *}$ & $5.672^{* * *}$ & $5.704^{* * *}$ \\
\hline & $(0.023)$ & $(0.059)$ & $(0.008)$ & $(0.029)$ & $(0.007)$ & $(0.023)$ \\
\hline $\mathrm{R} 2$ & 0.060 & 0.051 & 0.130 & 0.131 & 0.055 & 0.063 \\
\hline N. de Obs. & 501.962 & 37.140 & 501.962 & 37.140 & 501.962 & 37.140 \\
\hline
\end{tabular}

Fonte: Elaboração própria, a partir dos dados da Prova Brasil-SAEB (2017). Notas: ${ }^{*} p<0,1,{ }^{* *} p<$ $0,05,{ }^{* * *} p<0,01$ 
Tabela 2. FPE por quantis e área. Matemática - 2017

\begin{tabular}{|c|c|c|c|c|c|c|}
\hline & \multicolumn{2}{|c|}{ Q10 } & \multicolumn{2}{|c|}{ Q50 } & \multicolumn{2}{|c|}{ Q90 } \\
\hline & Urbano & Rural & Urbano & Rural & Urbano & Rural \\
\hline \multirow[t]{2}{*}{ Sexo al } & $0.028^{* * *}$ & $0.028^{* * *}$ & $0.059^{* * *}$ & $0.055^{* * *}$ & $0.042^{* * *}$ & $0.047^{* * *}$ \\
\hline & $(0.001)$ & $(0.004)$ & $(0.001)$ & $(0.003)$ & $(0.001)$ & $(0.003)$ \\
\hline \multirow[t]{2}{*}{ Idade } & $-0.04^{* * *}$ & $-0.03^{* * *}$ & $-0.016^{* * *}$ & $-0.02^{* * *}$ & $-0.004^{* * *}$ & $-0.01^{* * *}$ \\
\hline & $(0.001)$ & $(0.004)$ & $(0.001)$ & $(0.002)$ & $(0.0004)$ & $(0.001)$ \\
\hline \multirow[t]{2}{*}{ Mãe FC } & $0.021^{* * *}$ & 0.008 & $0.009^{* * *}$ & 0.004 & -0.001 & 0.003 \\
\hline & $(0.002)$ & $(0.006)$ & $(0.001)$ & $(0.003)$ & $(0.001)$ & $(0.004)$ \\
\hline \multirow[t]{2}{*}{ Mãe MC } & $0.032^{* * *}$ & $0.028^{* * *}$ & $0.024^{* * *}$ & $0.021^{* * *}$ & $0.010^{* * *}$ & $0.018^{* * *}$ \\
\hline & $(0.002)$ & $(0.005)$ & $(0.001)$ & $(0.004)$ & $(0.001)$ & $(0.004)$ \\
\hline \multirow[t]{2}{*}{ Mãe SC } & $0.018^{* * *}$ & 0.007 & $0.030^{* * *}$ & $0.018^{* * *}$ & $0.030^{* * *}$ & $0.045^{* * *}$ \\
\hline & $(0.002)$ & $(0.008)$ & $(0.001)$ & $(0.005)$ & $(0.001)$ & $(0.007)$ \\
\hline \multirow[t]{2}{*}{ Biblioteca } & $0.009^{* * *}$ & $0.009^{*}$ & $0.009^{* * *}$ & $0.015^{* * *}$ & $0.008^{* * *}$ & $0.012^{* * *}$ \\
\hline & $(0.002)$ & $(0.005)$ & $(0.001)$ & $(0.003)$ & $(0.001)$ & $(0.003)$ \\
\hline \multirow[t]{2}{*}{ Lab infor. } & $0.009^{* * *}$ & $0.024^{* * *}$ & $0.007^{* * *}$ & $0.021^{* * *}$ & $0.002^{* * *}$ & $0.011^{\text {**** }}$ \\
\hline & $(0.002)$ & $(0.004)$ & $(0.001)$ & $(0.003)$ & $(0.001)$ & $(0.003)$ \\
\hline \multirow[t]{2}{*}{ Inse } & $0.023^{* * *}$ & $0.025^{* * *}$ & $0.028^{* * *}$ & $0.024^{* * *}$ & $0.029^{* * *}$ & $0.020^{* * *}$ \\
\hline & $(0.001)$ & $(0.003)$ & $(0.001)$ & $(0.002)$ & $(0.001)$ & $(0.002)$ \\
\hline \multirow[t]{2}{*}{ Sexo prof. } & $-0.01^{* * *}$ & 0.007 & $-0.004^{* * *}$ & 0.0004 & $-0.002^{* *}$ & -0.001 \\
\hline & $(0.001)$ & $(0.004)$ & $(0.001)$ & $(0.003)$ & $(0.001)$ & $(0.003)$ \\
\hline \multirow[t]{2}{*}{ Faixa etária } & $-0.01^{* * *}$ & -0.002 & $-0.006^{* * *}$ & -0.003 & $-0.006^{* * *}$ & $-0.01^{* *}$ \\
\hline & $(0.001)$ & $(0.003)$ & $(0.001)$ & $(0.002)$ & $(0.001)$ & $(0.002)$ \\
\hline \multirow[t]{2}{*}{ Cor prof. } & $0.008^{* * *}$ & $0.020^{* * *}$ & $0.0095^{* * *}$ & $0.018^{* * *}$ & $0.008^{* * *}$ & $0.024^{* * *}$ \\
\hline & $(0.001)$ & $(0.005)$ & $(0.001)$ & $(0.003)$ & $(0.001)$ & $(0.004)$ \\
\hline \multirow[t]{2}{*}{ Lic. área } & $0.006^{* * *}$ & $0.017^{* * *}$ & $0.003^{* * *}$ & $0.017^{* * *}$ & $0.002^{* *}$ & $0.007^{* *}$ \\
\hline & $(0.002)$ & $(0.006)$ & $(0.001)$ & $(0.003)$ & $(0.001)$ & $(0.004)$ \\
\hline \multirow[t]{2}{*}{ Não tem pós } & $-0.01^{* * *}$ & -0.003 & $-0.007^{* * *}$ & 0.001 & $-0.007^{* * *}$ & 0.001 \\
\hline & $(0.001)$ & $(0.005)$ & $(0.001)$ & $(0.003)$ & $(0.001)$ & $(0.003)$ \\
\hline \multirow[t]{2}{*}{ Infraestrutura } & $-0.02^{* * *}$ & $-0.01^{* *}$ & $-0.02^{* * *}$ & $-0.02^{* * *}$ & $-0.017^{* * *}$ & $-0.02^{* * *}$ \\
\hline & $(0.001)$ & $(0.004)$ & $(0.001)$ & $(0.003)$ & $(0.001)$ & $(0.003)$ \\
\hline \multirow[t]{2}{*}{ Nordeste } & $0.006^{* *}$ & $0.017^{* * *}$ & $0.022^{* * *}$ & $0.036^{* * *}$ & $0.026^{* * *}$ & $0.036^{* * *}$ \\
\hline & $(0.003)$ & $(0.007)$ & $(0.001)$ & $(0.004)$ & $(0.001)$ & $(0.003)$ \\
\hline \multirow[t]{2}{*}{ Sudeste } & $0.025^{* * *}$ & $0.036^{* * *}$ & $0.036^{* * *}$ & $0.060^{* * *}$ & $0.018^{* * *}$ & $0.044^{* * *}$ \\
\hline & $(0.002)$ & $(0.008)$ & $(0.001)$ & $(0.005)$ & $(0.001)$ & $(0.006)$ \\
\hline \multirow[t]{2}{*}{ Sul } & $0.052^{* * *}$ & $0.040^{* * *}$ & $0.043^{* * *}$ & $0.062^{* * *}$ & 0.0004 & $0.020^{* *}$ \\
\hline & $(0.003)$ & $(0.010)$ & $(0.002)$ & $(0.006)$ & $(0.002)$ & $(0.008)$ \\
\hline Centro-Oeste & $0.046^{* * *}$ & $0.040^{* * *}$ & $0.040^{* * *}$ & $0.053^{* * *}$ & $0.014^{* * *}$ & $0.037^{* * *}$ \\
\hline
\end{tabular}




\begin{tabular}{lcccccc} 
& $(0.003)$ & $(0.010)$ & $(0.002)$ & $(0.007)$ & $(0.002)$ & $(0.009)$ \\
Constante & $5.613^{* * *}$ & $5.399^{* * *}$ & $5.561^{* * *}$ & $5.568^{* * *}$ & $5.676^{* * *}$ & $5.770^{* * *}$ \\
& $(0.020)$ & $(0.057)$ & $(0.009)$ & $(0.027)$ & $(0.008)$ & $(0.024)$ \\
\hline R2 & 0.056 & 0.043 & 0.140 & 0.138 & 0.072 & 0.077 \\
N. de Obs. & 516.461 & 38.523 & 516.461 & 38.523 & 516.461 & 38.523 \\
\hline
\end{tabular}

Fonte: Elaboração própria, a partir dos dados da Prova Brasil-SAEB (2017). Notas: ${ }^{*} p<0,1,{ }^{* *} p<$ $0,05,{ }^{* * *} p<0,01$

Os professores que têm licenciatura na área que lecionam influenciam positivamente no desempenho dos alunos nas duas disciplinas. O efeito é maior nas áreas rurais do que em áreas urbanas, contudo, a magnitude se reduz com o aumento do quantil. Nesse sentido, Vidal \& Vieira (2017) destacam que a maioria dos professores no Brasil que atuam em Língua Portuguesa e Matemática do ensino fundamental público possuem formação superior, porém nem sempre essa formação, ou pós-graduação, é na sua área de atuação. Portanto, é necessário fortalecer essa discussão e buscar soluções, uma vez que os estudantes podem melhorar seus desempenhos quando expostos a professores com formação adequada na área de atuação. Assim, o resultado encontrado corresponde com o esperado.

Quanto ao salário dos professores, o efeito positivo sobre o desempenho dos alunos corrobora evidências já encontradas, como em Zhang, Jin, Torero \& Li (2018). Maiores salários tendem a atrair professores mais qualificados e, consequentemente, o aprendizado dos alunos.

Tratando-se da localização regional dessas escolas, comparada à região Norte, o efeito é positivo. A única exceção ocorre para estudantes de baixo desempenho em escolas urbanas das regiões Nordeste e Sudeste, pois o efeito é negativo. Isto é, os resultados dos estudantes da região Norte, nesses casos, foram melhores. Além disso, o efeito da localização aumenta em quantis superiores e são maiores para as escolas da zona rural.

Vale destacar que para o desempenho dos alunos em Matemática, as variáveis utilizadas nos modelos não apresentaram comportamentos diferentes da situação apresentada para Língua Portuguesa. Além do efeito do sexo do estudante, outra exceção importante que difere sobre o desempenho em Português e Matemática são os efeitos da localização regional dessas escolas. Em Matemática, os efeitos regionais foram todos positivos, ou seja, alunos que estudam em outras regiões têm desempenhos positivos em Matemática quando comparados com a região Norte. 
Os resultados estimados para os efeitos regionais são condizentes com o esperado, isto é, as outras regiões do país apresentam melhores resultados educacionais do que a região Norte do Brasil. Historicamente, a região Norte, além de condições geográficas adversas, apresenta níveis de desenvolvimento inferior, o que dificulta a atração de professores mais qualificados. Ademais, os estudantes dessa região estão expostos a menores oportunidades no acesso à educação, especialmente na educação infantil. Estudantes da região Nordeste, que também apresenta desigualdades sociais relevantes, apresentaram melhores pontuações do que os estudantes do Norte do país, nos quantis 50 e 90.

Até o momento, foram analisadas as características que influenciam o desempenho dos estudantes em Língua Portuguesa e Matemática e como os efeitos se diferenciam entre áreas e nos quantis de desempenho. Na sequência, os resultados da decomposição são discutidos.

As estimativas das diferenças totais ${ }^{14}$ e explicadas do desempenho educacional dos alunos em cada quantil foram significantes a 5\%. À medida que aumentam os quantis, menor é a diferença de desempenho entre alunos de escolas urbanas e rurais, ou seja, a desigualdade é menor entre os alunos de alto desempenho.

No agregado, os diferenciais educacionais nas duas disciplinas são explicados principalmente pelas diferenças nas características observadas (componente explicado), uma vez que os alunos de escolas urbanas possuem mais dos insumos que afetam positivamente o desempenho do que os estudantes de escolas rurais. $\mathrm{O}$ efeito positivo do componente explicado, ao longo de toda distribuição, implica que se os alunos de escolas urbanas tivessem as mesmas características dos estudantes do meio rural, menores seriam suas pontuações. Esse resultado é compatível com os achados de Mattita \& Chirwa (2009) e Rodrigues et al. (2018). Ademais, percebe-se que a importância relativa do componente explicado se reduz entre os estudantes de baixo e alto desempenho em Língua Portuguesa, enquanto que em Matemática ocorre o contrário.

A Figura 1 apresenta, na parte superior, o efeito tangível total (componente explicado agregado), o efeito tangível puro e o erro de especificação. O termo de erro se aproxima de zero para as duas disciplinas, embora apresente algumas variações entre os quantis, como é esperado em distribuições funcionais não linea-

14 As estimativas da diferença total e da decomposição agregada nos quantis, para as duas disciplinas podem ser disponibilizadas pelos autores. 
res. Esses resultados mostram que o modelo é bem especificado e consistentemente estimado pelo modelo reponderado.

O efeito tangível detalhado para os cinco grupos de características é apresentado na parte inferior da Figura 1. Para todos os quantis, diferenças nas características da escola e da família são responsáveis por maior parte do efeito tangível.

As diferenças nas características dos professores, apesar de positivas, respondem pela menor parcela no componente. Isso sugere que se os estudantes de escolas urbanas fossem expostos a professores com as mesmas características dos professores de escolas rurais, eles teriam menor desempenho. Resultados similares foram encontrados para Matemática (Figura 1).

Os efeitos intangíveis para Língua Portuguesa e Matemática são apresentados na Figura 2. Na parte superior da figura de cada painel, pode ser observado os efeitos agregados das diferenças não explicadas (total e puro) e a diferença residual (erro de reponderação). Os efeitos não explicados puros foram positivos e não significantes em todos os quantis da distribuição. Os resultados sugerem que os alunos das escolas urbanas, no agregado dos coeficientes, têm maior capacidade de converter insumos educacionais em melhores resultados.

Na parte inferior da Figura 2 para cada disciplina é apresentada a decomposição detalhada dos efeitos intangíveis por grupo de características. Apenas a constante, as características dos alunos no quantil 60 e dos professores no 90 tiveram efeitos positivos. A constante é um componente que independe das variáveis utilizadas no modelo e é obtida pela diferença entre os interceptos urbanos e rurais. Segundo Lounkaew (2013), ela pode ser interpretada como a diferença de desempenho puro no quantil, que é explicada pelas diferenças nas características não observadas não incluídas no modelo. Os efeitos negativos no componente intangível para os grupos de características são compensados pelo efeito positivo da constante. Portanto, relevante é o efeito do intercepto (das características não observadas não incluídas no modelo) do componente não explicado para explicar o gap total.

Em outras palavras, o efeito intangível mede a mudança esperada no desempenho dos alunos de escolas rurais, caso eles tivessem os coeficientes dos discentes de escolas urbanas. No agregado, isto é, ao considerar os efeitos de todos os grupos de características, incluindo o intercepto, o efeito intangível foi positivo. Esse resultado sugere que, se estudantes de escolas rurais frequentassem o "ambiente escolar/ 
Figura 1. Decomposição do efeito tangível

\section{Língua Portuguesa}
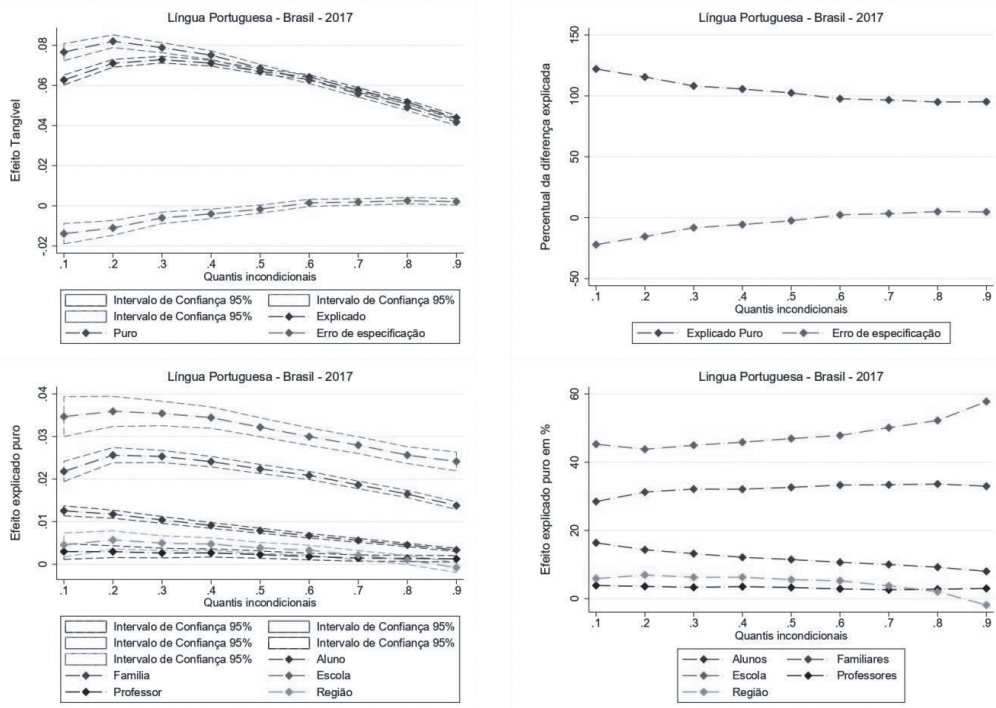

\section{Matemática}
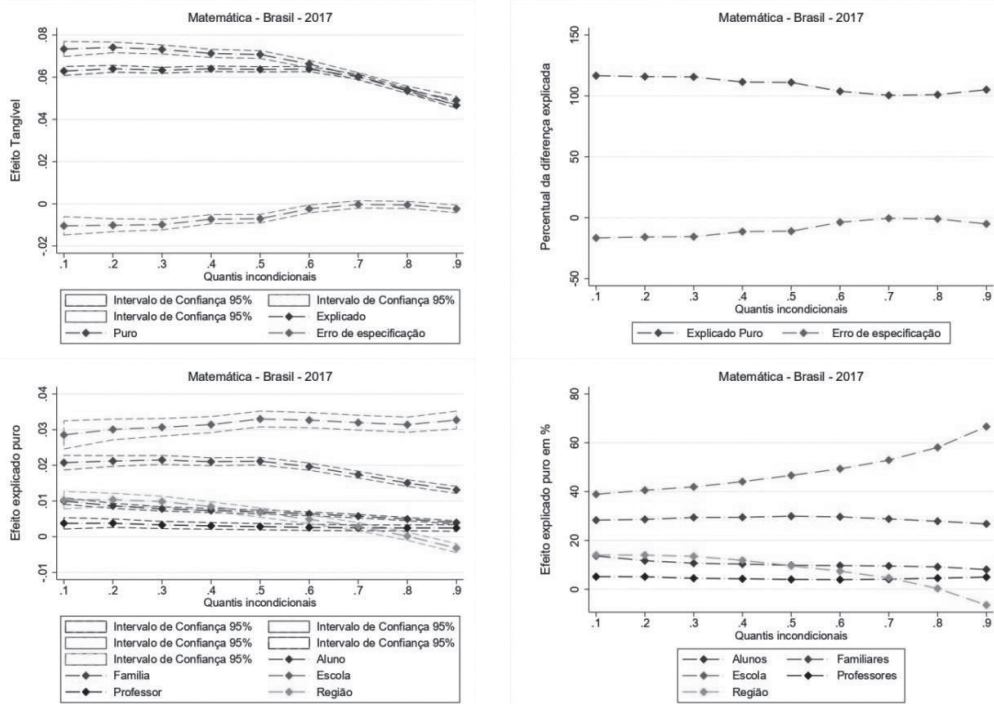

Fonte: Elaboração própria, a partir dos dados da Prova Brasil-SAEB (2017). 
Figura 2. Decomposição do efeito intangível

\section{Língua Portuguesa}
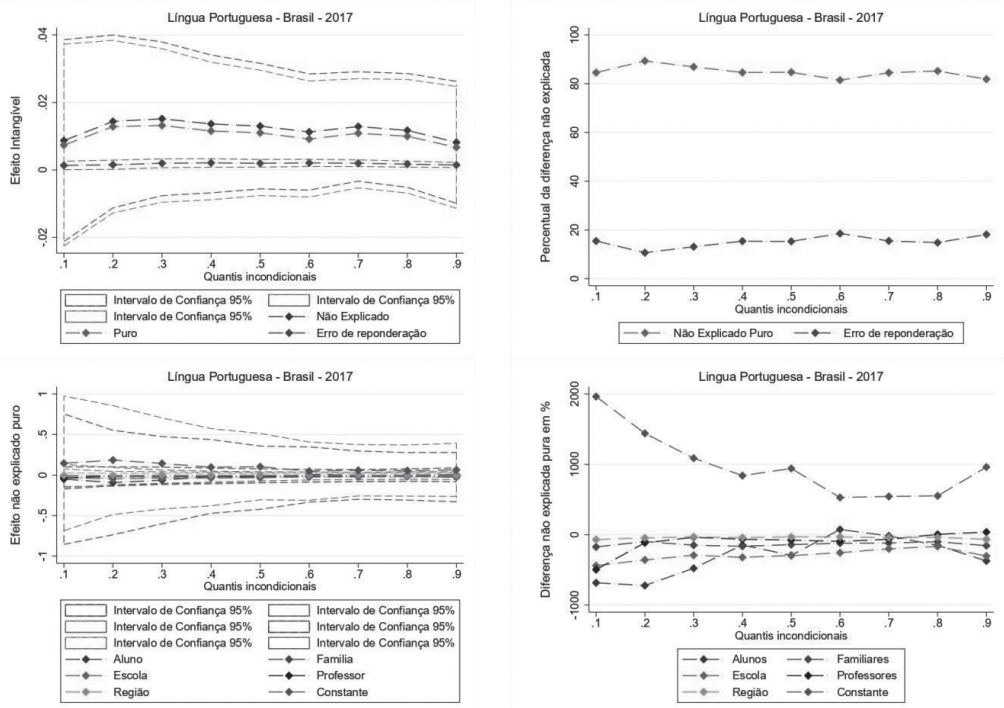

\section{Matemática}
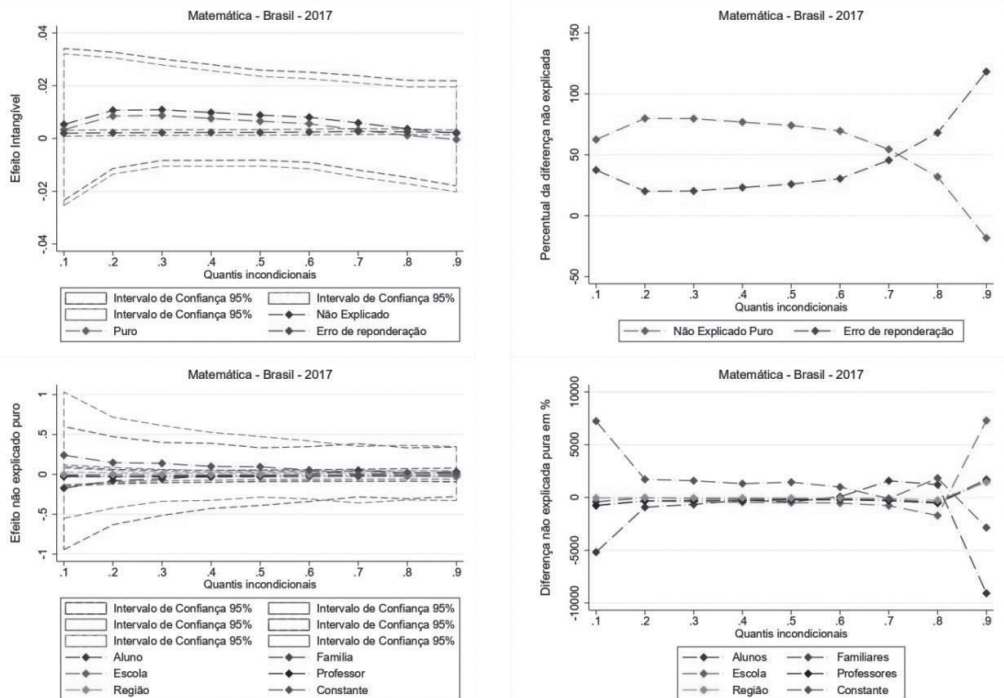

Fonte: Elaboração própria, a partir dos dados da Prova Brasil-SAEB (2017). 
educacional" dos estudantes de escolas urbanas, eles teriam melhores resultados. Contudo, esse resultado é atribuído ao efeito do positivo do intercepto, que supera os efeitos negativos das demais características. Desse modo, considerando apenas o efeito intangível das características observadas dos professores, isso não seria verdade. Ou seja, analisando apenas o efeito intangível dos professores, o desempenho dos estudantes de escolas rurais seria reduzido.

Os efeitos não explicados puros para Matemática também foram positivos e não significantes em todos os quantis da distribuição e semelhantes aos de Lingua Portuguesa. Contudo, existe uma diferença em relação aos estudantes de maior desempenho. Para esse grupo de estudantes, o efeito intangível foi negativo, sendo influenciado principalmente pelo efeito das características do aluno e da constante.

É importante ressaltar a relevância de analisar a distribuição de notas por quantis, pois possibilitam identificar os efeitos heterogêneos das características no hiato de notas entre as escolas urbanas e rurais brasileiras. Os resultados das covariáveis de forma detalhada na redução do hiato podem contribuir para identificar os fatores nos quais as políticas públicas poderiam agir para tentar solucionar essa desigualdade.

\section{CONCLUSÕES}

Os métodos utilizados no presente estudo possibilitaram a confirmação das diferenças existentes entre as áreas censitárias brasileiras e a contribuição dos docentes para o desempenho dos alunos. Professores que têm licenciatura na área influenciam positivamente no desempenho de seus alunos, o efeito é maior para os estudantes de escolas rurais e em ambas as áreas esse efeito reduz para alunos de maiores desempenhos.

Os resultados encontrados mostram que, para as estimativas dos diferenciais de desempenho que foram significantes, é possível destacar que os alunos das escolas urbanas apresentam vantagens em relação aos discentes de escolas rurais. Para as duas disciplinas, o diferencial total entre as notas dos alunos reduz à medida que aumenta o desempenho em proficiência, isto é, o diferencial é maior para aqueles com menor desempenho.

Verificou-se que o efeito tangível na maioria dos casos foi positivo e significante, portanto, se os alunos de escolas urbanas tivessem as mesmas características dos estudantes de escolas rurais, eles teriam menores desempenhos. Esse resultado 
é importante, pois aponta que é possível eliminar as desigualdades de rendimento dos alunos através de políticas públicas adequadas.

Os resultados da decomposição detalhada por grupos permitem observar que, enquanto os diferenciais das características escolares e familiares são importantes fatores que contribuem para a desigualdade educacional entre os alunos dessas escolas brasileiras, o efeito das características dos professores é menor. Por outro lado, nossos resultados também sugerem que se estudantes de escolas rurais frequentassem o ambiente educacional que os estudantes de escolas urbanas frequentam, os desempenhos desses estudantes seriam melhores, em função do efeito agregado do componente não explicado.

Espera-se que os alunos das escolas urbanas tenham maiores capacidade de converterem os insumos educacionais em melhores resultados, além de existirem outros fatores não observáveis que contribuem para os melhores desempenhos. Foram levantadas evidências que são os fatores tangíveis que contribuem para o aumento do gap. Mas, reduzir as disparidades nas características dos professores entre as duas áreas pode ser a solução menos eficaz para a redução dos diferenciais entre as duas áreas. Nesse sentido, políticas que ampliem a infraestrutura das escolas rurais, como a criação ou ampliação de bibliotecas, salas de estudo, laboratórios de informática etc., podem ser mais eficazes para esse objetivo. Além disso, ampliação dos investimentos em transporte escolar e nas estradas para que o acesso às escolas urbanas por parte de estudantes que residem no meio rural pode ser outra opção para reduzir o gap.

Mesmo que historicamente estejam presentes avanços em relação às avaliações, ainda existe a necessidade do uso dos dados disponíveis, para formulação de políticas mais eficientes de combate às disparidades educacionais, possibilitando então um sistema mais igualitário.

\section{REFERÊNCIAS}

Alves, M. T. G., \& Soares, J. F. (2013). Contexto escolar e indicadores educacionais: condições desiguais para a efetivação de uma política de avaliação educacional. Educação e Pesquisa (USP. Impresso), 39, 177-194.

Andrade, M., Franco, C., \& Carvalho, J. P. (2003). Gênero e desempenho em matemática ao final do ensino médio: quais as relações? Estudos em Avaliação Educacional, 27, 77-96. 
Angrist, J. D., \& Lavy, V. (1999). Using maimonides'rule to estimate the effect of class size on scholastic achievement. The Quarterly Journal of Economics, 114(2), 533-575.

Blinder, A. S. (1973). Wage discrimination: Reduced form and structural estimates. Journal of Human Resources, 8(4), 436-455.

Byun, S. Y., Meece, J. L., \& Irvin, M. J. (2012). Rural-nonrural disparities in postsecondary educational attainment revisited. American educational research journal, 49(3), 412-437.

Clotfelter, C. T., Ladd, H. F., \& Vigdor, J. L. (2007). Teacher credentials and student achievement: Longitudinal analysis with student fixed effects. Economics of Education Review, 26(6), 673-682.

Dinardo, J., Fortin, N. M., \& Lemieux, T. (1996). Labor Market Institutions and the Distribution of Wages, 1973-1992: A Semiparametric Approach. Econometrica, 64, 1001-1044.

Entorf, H., \& Minoiu, N. (2005). What a difference immigration policy makes: a comparison of PISA scores in Europe and traditional countries of immigration. German Economic Review, 6(3), 355-376.

Firpo, S. P., Fortin, N. M., \& Lemieux, T. (2018). Decomposing Wage Distributions Using Recentered Influence Function Regressions. Econometrics, 6 (2), 1-40. doi: doi.org/10.3390/econometrics6020028

Fortin, N. M., Lemieux, T., \& Firpo, S. (2011). Decomposition methods in economics. Handbook of Labor Economics, 4 (1), 1-102.

Hammond, L. D. (2000). Teacher quality and student achievement. Education policy analysis archives, 8 (1), 1-44. Disponivel em https://doi.org/10.14507/ epaa.v8n1.2000

Hanushek, E. A. (1970). The production of education, teacher quality and efficiency. Do Teachers Make a Difference? Washington, DC: Government Printing Office, 79-99. Disponível em https://eric.ed.gov/?id=ED037396

INEP, Instituto Nacional de Estudos e Pesquisas Educacionais Anísio Teixeira. (2020). Dados Abertos. Disponível em: http://inep.gov.br/web/guest/dados

Krueger, A. (1999). Experimental Estimates of Education Production Functions. Quarterly Journal of Economics, 114(2), pp.497-532.

Lounkaew, K. (2013). Explaining urban-rural differences in educational achievement in Thailand: Evidence from PISA literacy data. Economics of Education Review, 37(10), 213-225. Disponível em: https://doi.org/10.1016/j. econedurev.2013.09.003

Marks, G. N. (2006). Family size, family type and student achievement: Cross-national differences and the role of socioeconomic and school factors. Journal of Comparative Family Studies, 37(1), 1-24. 
Mattita, M. M., \& Chirwa, E. W. (2009). Rural-urban inequalities in malawi: Evidencce from a decomposition analysis. Working paper, University of Malawi Chancellor College Department of Economics. Disponível em: http://wadonda.com/wp2009_05.pdf

Menezes-Filho, N. A., \& Pazello, E. (2007). Do teachers' wages matter for proficiency? Evidence from funding reform in Brazil. Economics of Education Review, 26(6), 660-672. Disponível em https://doi.org/10.1016/j.econedurev.2007.08.003

Oaxaca, R. L. (1973). Male-female differentials in urban labor market. International Economic Review, Pennsylvania, 14 (3), 693-709. doi: 10.2307/2525981

Pessôa, S. A., \& Barbosa Filho, F. H. (2008). Retorno da Educação no Brasil. Pesquisa e Planejamento Econômico, Rio de Janeiro, 38, 97-125. Disponível em: ppe.ipea.gov.br/index.php/ppe/article/viewFile/130/1024

Ramos, R., Duque, J. C., \& Nieto, S. (2016). Decomposing the Rural-Urban Differential in Student Achievement in Colombia using PISA Microdata. Estudios de Economía Aplicada, 34 (2), 379-411. Disponível em https://www. redalyc.org/pdf/301/30146038005.pdf

Rodrigues, L., Costa, E., Miro, V., Mariano, Z., \& Jesus Filho, J. (2018). Determinants of the differential in schooling performance between urban and rural schools in Brazil. Em: XXI Encontro de Economia da Região Sul - ANPEC/ SUL, Curitiba-PR. Disponível em https://www.anpec.org.br/sul/2018/submissao/files_I/i2-2b3ce3fe4250blea23f885e3849779dd.pdf

Rodrigues, L., Costa, E., Miro, V., \& Mariano, Z. (2017). Migração educacional rural-urbana no Nordeste brasileiro: uma análise do diferencial de desempenho escolar no ensino médio. In: XV Encontro Nacional da Associação Brasileira de Estudos Regionais e Urbanos. São Paulo-SP.

Roscigno, V. J., \& Crowley, M. L. (2001). Rurality, institutional disadvantage, and achievement/ attainment. Rural Sociology, 66, 268-292. doi: doi. org/10.1111/j.1549-0831.2001.tb00067.x

Roscigno, V. J., Tomaskovic-Devey, D., \& Crowley, M. (2006). Education and the inequalities of place. Social Forces, 84 (4), 2121- 2145. Disponível em https://doi.org/10.1353/sof.2006.0108

Sakellariou, C. (2012). Decomposing the increase in TIMSS Scores in Ghana: 2003-2007 (English). Policy Research Working paper (No. WPS 6084). Washington, D.C.: World Bank Group. Disponível em http://documents. worldbank.org/curated/en/705881468031495601/Decomposing-theincrease-in-TIMSS-Scores-in-Ghana-2003-2007

Strayhorn, T. L. (2009). Different Folks, Different Hopes: The Educational Aspirations of Black Males in Urban, Suburban, and Rural High 
Schools. Urban Education, 44 (6), 710-731. Disponível em https://doi. org/10.1177/0042085908322705

Tayyaba, S. (2012). Rural-urban gaps in academic achievement, schooling conditions, student, and teachers' characteristics in Pakistan. International Journal of Educational Management, 26 (1), 6-26.

Vidal, E. M., \& Vieira, S. L. (2017). Professores da educação básica: perfil e percepções sobre sucesso dos alunos. Estudos em Avaliação Educacional (Online), 67, 64-101.

Wang, J., Li, H., \& Wang, D. (2018) Bridging the Rural-Urban Literacy Gap in China: A Mediation Analysis of Family Effects. Journal of research in childhood education, 32 (1), 119-134.

Wang, D., Wang, J., Li, H., \& Li, L. (2017). School context and instructional capacity: A comparative study of professional learning communities in rural and urban schools in China. International Journal of Educational Development, 52, 1-9. Disponível em https://doi.org/10.1016/j.ijedudev.2016.10.009

Woessmann, L. (2010). Families, schools, and primary-school learning: Evidence from Argentina and Colombia in an international perspective. Applied Economics, 42(21), 2645-2665. Disponível em https://doi. org/10.1080/00036840801964617

Yun, M. S. (2005). A Simple Solution to the Identification Problem in Detailed Wage Decompositions. Economic Inquiry, 43 (4), 766-772. Disponível em https://doi.org/10.1093/ei/cbi053

Zhang, J., Jin, S., Torero, M., \& Li, T. (2018). Teachers and urban-rural gaps in educacional outcomes. American Journal of Agricultural Economics, 100 (4), 1207-1223. Disponível em https://doi.org/10.1093/ajae/aay009

(C) 2021 por los autores; licencia no exclusiva otorgada a la revista Estudios económicos. Este artículo es de acceso abierto y distribuido bajo los términos y condiciones de una licencia Atribución-No Comercial 4.0 Internacional (CC BY-NC 4.0) de Creative Commons. Para ver una copia de esta licencia, visite http://creativecommons.org/licenses/by-nc/4.0 